\title{
A GENERAL DEPENDENCE RELATION FOR LATTICES ${ }^{1}$
}

\section{DANIEL T. FINKBEINER}

1. Introduction. An arithmetic theory of lattices, which concerns the representation of lattice elements in terms of irreducible elements, has been developed only for lattices which satisfy the semi-modular law and a chain condition. For such a lattice $\mathfrak{l}$ the principal results are:

(1) $\mathfrak{R}$ has unique irredundant decompositions if and only if $\mathfrak{R}$ is locally distributive $[1] .^{2}$

(2) The number of components in an irredundant decomposition is unique if and only if $\mathfrak{R}$ is locally modular [2].

However, the uniqueness of the number of components does not imply semi-modularity, and a characterization of lattices which have this uniqueness property will require an arithmetic theory for a more extensive class of lattices.

A natural method of approach to such a theory is to imbed an arbitrary lattice in a semi-modular lattice. However, the usual method of imbedding by means of dependence relations produces lattices in which the join irreducibles are points and the meet irreducibles are maximal elements. Clearly this is not suitable for arithmetic considerations. The purpose of this note is to formulate the properties a dependence relation must have if the imbedding is to preserve join irreducibility and if the imbedding lattice is to be semimodular. Since the join irreducibles form a partially ordered set $Q$, the dependence relation will be defined on subsets of $Q$.

2. Terminology and notation. The lattice theoretic terms used here are standard [3]. Unless otherwise noted, irreducible means joinirreducible. The null element is trivially irreducible and is excluded from sets of irreducibles. Lattice operations are denoted $U$ and $\cap$, and the corresponding set operations by $\vee$ and $\wedge$. If $q$ is an element of a partially ordered set $Q$, let $S_{q}=\left\{q^{\prime} \in Q \mid q^{\prime} \subseteq q\right\}$.

3. Properties of the dependence relations. A relation $D$ between the elements and subsets of a set $Q$ is called a dependence relation

Presented to the Society, December 29, 1949; received by the editors November 22, 1950.

${ }_{1}^{1}$ This note constitutes part of a thesis submitted to the California Institute of Technology. The author is grateful to Professor R. P. Dilworth for his many suggestions.

2 Numbers in brackets refer to the references at the end of the paper. 
provided $D$ satisfies

(D1) $q D S \vee q$ for arbitrary $S \subseteq Q$,

(D2) $q D S$ and $S D T$ implies $q D T .^{3}$

The closure of $S$ is defined by $C(S)=\{q \in Q \mid q D S\}$. The closed subsets of $Q$ form a complete lattice $\mathcal{R}^{\prime}$, and $\mathcal{R}^{\prime}$ is a semi-modular point lattice provided $D$ also satisfies the exchange axiom

(D3) $q D S \vee q^{\prime}$ implies either $q D S$ or $q^{\prime} D S \vee q[4]$

Now let $Q$ be a partially ordered set, and let $\Delta$ be a relation between the elements and subsets of $Q$. The following properties of $\Delta$ are considered.

$(\Delta 1) q^{\prime} \subseteq q$ implies $q^{\prime} \Delta S \vee q$ for arbitrary $S \subseteq Q$.

$(\Delta 2) q \Delta S$ and $S \Delta T$ implies $q \Delta T$.

( $\Delta 3) q^{\prime} \Delta q$ implies $q^{\prime} \subseteq q$.

$(\Delta 4) q \Delta S$ and $S \Delta q$ implies $q \in S$.

$(\Delta 5)$ If $q^{\prime \prime} \subset q^{\prime}$ implies $q^{\prime \prime} \Delta S$, then $q \Delta S \vee q^{\prime}$ implies either $q \Delta S$ or $q^{\prime} \Delta S \vee q$.

Any relation which satisfies $\Delta 1$ and $\Delta 2$ is a dependence relation, and the closed sets form a complete lattice $\mathfrak{R}^{\prime}$. The following lemmas are proved readily.

LEMma 1. If $\Delta$ satisfies $\Delta 1-\Delta 3$, then $S_{q}$ is closed for all $q \in Q$.

LEMMA 2. If $\Delta$ satisfies $\Delta 1-\Delta 3$ and if $S$ is completely irreducible in $\mathfrak{R}^{\prime}$, then $S=S_{q}$ for some $q \in Q$.

LEMмA 3. If $\Delta$ satisfies $\Delta 1-\Delta 3, S_{q}$ is completely irreducible in $\mathfrak{R}^{\prime}$ for every $q \in Q$ if and only if $\Delta 4$ is also satisfied.

The first lemma implies that the natural mapping $q \rightarrow S_{q}$ is an imbedding of $Q$ into $\mathfrak{R}^{\prime}$. Clearly $S_{q^{\prime}} \subseteq S_{q}$ if and only if $q^{\prime} \subseteq q$, and if equality holds in either relation, it holds in both. This gives the following result.

THEOREM 1. Let $\Delta$ satisfy $\Delta 1-\Delta 3$. The set $Q^{\prime}$ of completely irreducible elements of $\mathfrak{R}^{\prime}$ is isomorphic to $Q$ under the natural mapping if and only if $\Delta 4$ is also satisfied.

Now consider the question of semi-modularity. MacLane [4] has formulated an exchange axiom (E6) which is free from any covering assumptions and which implies semi-modularity.

(E6) If $S \cap T \subset R \subset T \subset S \cup T$, then $S_{1}$ exists such that $S \cap T \subset S_{1}$ $\subseteq S$, and $\left(R \cup S_{1}\right) \cap T \subset T$.

Theorem 2. If $\Delta$ satisfies $\Delta 1, \Delta 2$, and $\Delta 5$ and if $Q$ satisfies the de-

\footnotetext{
\& By $S D T$ we mean $q D T$ for all $q \in S$.
} 
scending chain condition, then $\mathfrak{R}^{\prime}$ satisfies E6 and therefore is semimodular.

Proof. Let $S, T$, and $R$ be closed sets such that $S \cap T \subset R \subset T$ $C S \cup T$. The descending chain condition implies the existence of $q_{s}^{\prime} \in S-S \wedge T$ such that $q^{\prime \prime} \subset q_{s}^{\prime}$ implies $q^{\prime \prime} \Delta S \wedge T$. Clearly $q^{\prime \prime} \Delta R$ for all $q^{\prime \prime} \subset q_{s}^{\prime}$. Let $q_{t} \in T-R$; then $q_{t} \bar{\Delta} R .^{4}$ If $q_{t} \Delta R \bigvee q_{s}^{\prime}$, then by $\Delta 5$ $q_{s}^{\prime} \Delta R \bigvee q_{t}$, and therefore $(S \wedge T) \vee q_{s}^{\prime} \Delta R \bigvee q_{t}$. Let $S_{1}=C\left[(S \wedge T) \bigvee q_{s}^{\prime}\right]$ $\subseteq S$. Then $S_{1} \subseteq C\left(R \vee q_{t}\right) \subseteq T$, and $S_{1} \subseteq S \wedge T$ which contradicts the choice of $q_{s}^{\prime}$. Hence for all $q_{t} \in T-R, q_{t} \bar{\Delta} R \bigvee q_{s}^{\prime}$ and $C\left(R \vee q_{s}^{\prime}\right) \wedge T \subset T$. But also $C\left(R \bigvee S_{1}\right)=C\left(R \bigvee q_{8}^{\prime}\right)$, and finally $\left(R \cup S_{1}\right) \cap T \subset T$.

It should be observed that Theorem 2 implies the classical theorem concerning dependence relations and semi-modular point lattices [4] when $Q$ is an unordered set. Theorems 1 and 2 combine to give the main result.

THEOREM 3. Let $Q$ be a partially ordered set in which the descending chain condition holds. If $\Delta$ satisfies $\Delta 1-\Delta 5$, the lattice $\mathfrak{R}^{\prime}$ of closed subsets of $Q$ is a complete semi-modular lattice. The set of completely irreducible elements of $\mathfrak{R}^{\prime}$ is isomorphic to $Q$, and every element of $\mathfrak{R}^{\prime}$ is the union of such elements.

As an example, let $Q$ be the set of irreducible elements of a lattice which satisfies the descending chain condition. Define the relation

(A) $q \Delta S$ if and only if $q \subseteq \cup S$.

It is easy to verify that (A) satisfies $\Delta 1-\Delta 4$, and that the mapping $b \rightarrow S_{b}$ imbeds $\mathfrak{R}$ isomorphically onto $\mathfrak{R}^{\prime}$. If (A) satisfies $\Delta 5, \mathfrak{R}^{\prime}$ is semimodular; hence so is $\mathbb{R}$. The converse is also true; if $\mathfrak{R}$ is semi-modular, (A) satisfies $\Delta \mathbf{5}$.

It is interesting to note that some restriction of $Q$, such as the chain condition, is necessary in order that $\Delta \mathbf{5}$ imply the semi-modularity of $\mathfrak{R}^{\prime}$. Consider the lattice defined by

$$
\begin{aligned}
& u>t>s>q_{1}>q_{2}>\cdots \supset z, \\
& u>r>p_{1}>p_{2}>p_{3}>\cdots \supset z, \\
& u>r>q_{1},
\end{aligned}
$$

where every element except $u$ and $r$ is completely irreducible. Relation (A) satisfies $\Delta 1-\Delta \mathbf{5}$ when defined on $\mathbb{R}$, but $\mathbb{R}^{\prime}$ is isomorphic to $\mathbb{R}$ and is not semi-modular.

Other specific examples of irreducibility-preserving dependence re-

\footnotetext{
$4 \bar{\Delta}$ denotes the denial of $\Delta$.
} 
lations which have been applied to lattice imbedding problems will be given elsewhere.

\section{REFERENCES}

1. R. P. Dilworth, Lattices with unique irreducible decompositions, Ann. of Math. vol. 41 (1940).

2. - The arithmetical theory of Birkhoff lattices, Duke Math. J. vol. 8 (1941).

3. G. Birkhoff, Lattice theory, Amer. Math. Soc. Colloquium Publications, vol. 25, rev. ed., 1949.

4. S. MacLane, $A$ lattice formulation for transcendence degrees and p-bases, Duke Math. J. vol. 4 (1938).

California Institute of Technology and

YALE UNIVERSITY 